\title{
LA FORMACIÓN INTEGRAL DEL MÉDICO PARA ENFRENTAR LAS BARRERAS PARA LA ATENCIÓN COMUNITARIA A PROPÓSITO DE EL MÉDICO AFRICANO
}

\author{
Integral medical education to face barriers for community healthcare in \\ The African physician
}

Isaac E. AGUILAR-CANTÓN, Leydi G. BURGOS-MONTERO, Lucero CAÑEDO-AMAYA, Jorge C. GUILLERMO-HERRERA, Josseph E. UC-VÁZQUEZ, Nina MENDEZ DOMINGUEZ

Escuela de Medicina. Universidad Marista de Mérida (México).

Autora para correspondencia: Nina Méndez Domínguez.

Correo electrónico: ninuxka@hotmail.com

Recibido: 7 de octubre de 2020

Aceptado: 26 de diciembre de 2020

\section{Resumen}

El conjunto de conocimientos, habilidades, principios y valores de un individuo para ejercer bien su trabajo en medicina sirven para fortalecer el desenvolvimiento en una comunidad. La película Bienvenue à Marly-Gomont (2016) de Julien Rambaldi, tiene por tema principal el esfuerzo y las actitudes del médico congoleño Zantoko ante el racismo que demuestran los habitantes de la villa rural Marly-Gomont de los años setenta. La película muestra a una comunidad francesa aislada, quienes nunca habían visto a una persona de color; nos sitúa con la familia de Zantoko en el contexto de la comunidad, lo que nos permite comprender los puntos fundamentales que pudieron haber servido como herramientas prácticas y necesarias para la labor del médico comunitario. Nos invita a reflexionar acerca de las dificultades que pueden presentarse ante la falta de conocimientos necesarios para atender una comunidad y la poca prevención en dicha comunidad. En suma, es una película excelente para la enseñanza y aprendizaje de temas relacionados con la labor que desarrollan los profesionales de la salud a nivel poblacional/local.

Palabras clave: salud pública; educación médica; atención primaria; racismo; aprendizaje y responsabilidad social. 


\section{LA FORMACIÓN INTEGRAL DEL MÉDICO PARA ENFRENTAR LAS BARRERAS PARA LA ATENCIÓN COMUNITARIA A PROPÓSITO DE EL MÉDICO AFRICANO}

ISAAC E. AGUILAR-CANTÓN, LEYDI G. BURGOS-MONTERO, LUCERO CAÑEDO-AMAYA, JORGE C. GUILLERMO-HERRERA, JOSSEPH E. UC-VÁZQUEZ, NINA MENDEZ DOMINGUEZ

\section{Summary}

An individual's set of knowledge, skills, principles and values to carry out his medical work well serves to strengthen the unfolding in a community. The film the African physician (2016) of Julien Rambaldi, has as its main theme the effort and attitudes of the Congolese doctor Zantoko and how he faces the fear racism of the habitans of rural village Marly-Gomont on the seventies. The film shows an isolated French community, who had never seen black people; places us with Zantoko's family in the context of the community, which allows us to understand the fundamental point that may have served as practical and necessary tools for the work of the community doctor. It invites us to reflect on the difficulties that may occur in the face of the lack of knowledge needed to serve a community and ignorance of it. It is an excellent film for teaching and learning topics related to the work of health professionals at the population/local level.

Keywords: public health; medical education; primary care; racism; learning and social accountability.

\section{Ficha técnica}

Título: El médico africano.

Título original : Bienvenue à Marly-Gomont.

País: Francia.

Año: 2016.

Director: Julien Rambaldi.

Música: Emmanuel Rambaldi.

Fotografía: Yannick Ressigeac.

Montaje: Bruno Amestoy.

Guion: Benoît Graffin, Kamini Zantoko, Julien Rambaldi.

Intérpretes: Marc Zinga, Aïssa Maiga, Jonathan Lambert, Rufus Magloire, Nissim Renard, Jean-Benoît Ugeux, Médina Diarra, Stéphane Bissot.

Color: color.

Duración: 96 minutos.

Género: comedia (comedia dramática).

Idioma original: francés.

Sinopsis: “En 1975, Seyolo Zantoko, recién graduado como médico originario de Kinshasa, acepta una vacante de médico rural en un pequeño pueblo francés. Cuando llega a Marly-Gomont, la gente tiene miedo porque nunca han visto a una persona de raza negra, pero Seyolo está decidido a luchar y hará todo lo posible para ganarse la confianza de los aldeanos" (FILMAFFINITY).
Productoras: E.D.I Films, Curiosa Films,Moana Films ,TF1 Films Production, Scope Pictures, La Wallonie, Bruxelles Capitale, Canal+ , TF1 y Cinéfrance Plus.

\section{Enlaces :}

https://www.imdb.com/title/tt5555502/

https://www.filmaffinity.com/es/film447499. html

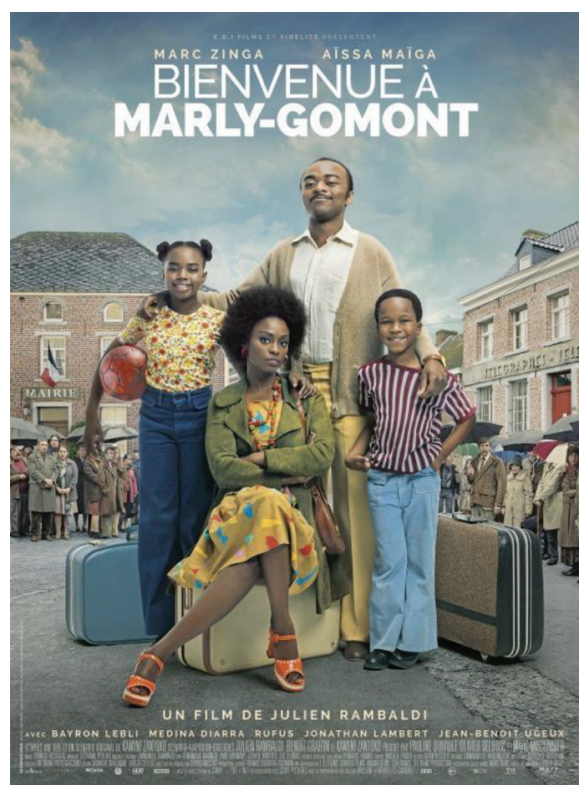




\section{LA FORMACIÓN INTEGRAL DEL MÉDICO PARA ENFRENTAR LAS BARRERAS PARA LA ATENCIÓN COMUNITARIA A PROPÓSITO DE EL MÉDICO AFRICANO}

ISAAC E. AGUILAR-CANTÓN, LEYDI G. BURGOS-MONTERO, LUCERO CAÑEDO-AMAYA, JORGE C. GUILLERMO-HERRERA, JOSSEPH E. UC-VÁZQUEZ, NINA MENDEZ DOMINGUEZ

\section{Introducción}

Bienvenue à Marly-Gomont (2016) del director Julien Rambaldi, enmarca los retos que afrontan los médicos al ser enviados para cumplir sus labores en los servicios de atención primaria en las comunidades, de igual forma resalta la importancia del compromiso con la sociedad independientemente de la situación que se presente durante la prestación de servicios médicos.

Seyolo Zantoko, egresado en medicina de la universidad francesa, decide ejercer su profesión en una comuna de Francia, Marly-Gomont, que necesitaba de manera urgente un médico. En la película podemos observar el conflicto que enfrenta Zantoko al migrar de la ciudad al campo para ejercer por primera vez como médico en una comunidad y ante el cambio de contexto social; a pesar de su optimismo no consideró que los habitantes por sus rasgos culturales y estereotipos preferían consultar con otro doctor que estaba a 15 kilómetros más lejos, desplazando con ello su trabajo.

La participación social en salud es un proceso complejo que conduce al cumplimiento de metas comunitarias, crecimiento personal y mejora entre los involucrados ${ }^{1}$, de modo que, si la comunidad no accede a la atención médica, ésta será deficiente en lograr su objetivo; por lo que existe la necesidad de implementar medidas que promuevan los servicios que el médico brinda.

La atención primaria al estar ligada directamente con la salud pública y medicina preventiva tiene su enfoque en la construcción de nuevas propuestas epistemológicas orientadas al desarrollo sustentable y continuo de la comunidad, considerando a las personas como entes biopsico-sociales, por lo que no solo se busca la rehabilitación de la salud, sino que también se centra en la prevención y promoción de la misma con el fin de preservarla².

La primera vez que vemos a Zantoko laborando como médico (Foto 1) es cuando llega a su consultorio una mujer embarazada preguntando por el nuevo doctor y ésta le rechaza la atención médica al darse cuenta que la examinaría un médico de color; la población no estaba al tanto de la llegada de un médico de otra etnia a la comunidad, las diferencias significativas de las particularidades físicas como el sexo, el color y la raza del médico entre las características biológicas de la comunidad logra ser proporcional al grado de empatía/interés que puede sentir el paciente debido a que, los estereotipos y prejuicios marcan una distancia interpersonal haciéndolos forzar en una línea definida por su etnia, dificultando el contacto e intercambio de información para su convivencia ${ }^{3}$.

El objetivo del presente escrito consiste en presentar la importancia de las estrategias educativas y las fortalezas que debe adquirir el médico en su formación ética, humana y profesional, así mismo analizar la conducta del doctor Zantoko ante la discriminación que enfrentó con respecto a las características de la comunidad Marly-Gomont.

\section{La medicina comunitaria y la comunidad francesa}

El origen de la medicina comunitaria nace de la evidencia directa o indirecta de que las enfermedades dependen en su mayoría, de condiciones ambientales no propicias (mal abastecimiento de agua, drenaje defectuoso, comida impura, entorno laboral desfavorable, entre otros) que se dan en los pequeños sectores de la población. En el siglo XIX tras la mejora de los aspectos sanitarios, es notoria la disminución de la incidencia de ciertas enfermedades a pesar de la falta de tratamiento médico específico ${ }^{4}$.

Con el constante crecimiento poblacional que acarrea consigo la presión por atender las necesidades de las comunidades (enfermedades, cuidados, tratamiento, costos) surge la obligación de crear la medicina comunitaria para atender a la población mediante la atención primaria (promoción/prevención) de esta forma reducir 


\section{LA FORMACIÓN INTEGRAL DEL MÉDICO PARA ENFRENTAR LAS BARRERAS PARA LA ATENCIÓN COMUNITARIA A PROPÓSITO DE EL MÉDICO AFRICANO}

ISAAC E. AGUILAR-CANTÓN, LEYDI G. BURGOS-MONTERO, LUCERO CAÑEDO-AMAYA, JORGE C. GUILLERMO-HERRERA, JOSSEPH E. UC-VÁZQUEZ, NINA MENDEZ DOMINGUEZ

los gastos y optimizar la calidad de vida en una población determinada 5 .

La evidencia identifica la medicina comunitaria como una disciplina originada en E.E.U.U. a partir de la década de 1960 como un modelo para combatir la pobreza. En sus inicios, fue llevada a cabo por instituciones voluntarias y asociaciones estatales y posteriormente incorporada en las escuelas médicas desde la perspectiva de la medicina preventiva ${ }^{5}$.

En el año de 1978, tras la conferencia de Alma Ata, surgió la medicina comunitaria como un área de especialidad médica. La Organización Mundial de la Salud (OMS) hizo oficial el resurgimiento de la clásica medicina general y le dio una nueva dimensión, más allá de la clínica y promoviendo el desarrollo del paradigma del paciente como un ser biopsicosocial'.

En el contexto de Francia en la década de 1970, (aunque no muy diferente a la actualidad) los médicos franceses no se instalaban en zonas rurales o en áreas periféricas de las grandes ciudades porque no eran rentables, pero el doctor Zantoko, con el propósito de adquirir la nacionalidad francesa decidió ser entrevistado por el alcalde de la comunidad y tener la oportunidad de adquirir el puesto de médico general de Marly-Gomont.

El sistema de salud francés pertenece a los sistemas bismarckianos (sistema de alianzas internacionales). El derecho a la asistencia sanitaria en Francia está ligado a la condición del trabajador y el salario médico está sujeto a la actividad del mismo ; por lo que, como se observa en la película, el doctor Zantoko tuvo que encontrar un trabajo temporal en una granja de la comunidad para solventar los gastos familiares; en ausencia de pacientes que atender, por consiguiente se puede relacionar estas actitudes (miedo a él y su familia) como un nivel de racismo a quienes eran desconocidos en Marly-Gomont.

Francia cuenta con una serie de características que lo convierten en blanco de discriminación en los entornos sanitarios; es el país con la segunda población más grande de inmigrantes nacidos fuera de la unión europea, siendo mayormente provenientes del norte de África, el sur de Europa y China ${ }^{8}$. Se contrastó que una característica del racismo es que su estructura e ideología pueden persistir en las políticas gubernamentales (como el candidato a la alcaldía quien decía calumnias sobre Zantoko) e institucionales, en ausencia de actores individuales que tengan prejuicios raciales explícitos ${ }^{9}$, esta visión conduce a actitudes negativas (prejuicios) hacia los grupos raciales (etnia africana de la familia Zantoko).

La discriminación influye en la salud de manera indirecta; en Francia, el estado no reconoce distinción étnica ni racial, por lo que las minorías no son consideradas objetivos en las políticas sociales, resultando en conductas que impiden el correcto abordaje en la interacción medico paciente. Se han documentado disparidades en las tasas de discriminación en la atención médica por la raza, etnia, estatus de inmigrante, dominio del idioma y estatus de seguro ${ }^{9}$. Es importante mencionar que la crisis social y económica en Francia ha acentuado una distribución geográfica desigual en todos los niveles de salud debido a que, el acceso a la atención médica disminuye en zonas remotas o pobres para acceder a la misma ${ }^{10}$.

La medicina comunitaria del siglo XXI es el producto de un proceso evolutivo prolongado, lo que sugiere que, tanto en los orígenes de la medicina como en su práctica, se ha desarrollado una visión más amplia del papel del médico en la comunidad.

\section{Formación integral del médico y la medicina comunitaria}

La medicina comunitaria requiere la integración de conocimientos pertenecientes a las ciencias sociales para lograr el bien colectivo; las acciones y características que se requieren para alcanzar el objetivo implican conocer los 


\section{LA FORMACIÓN INTEGRAL DEL MÉDICO PARA ENFRENTAR LAS BARRERAS PARA LA ATENCIÓN COMUNITARIA A PROPÓSITO DE EL MÉDICO AFRICANO}

ISAAC E. AGUILAR-CANTÓN, LEYDI G. BURGOS-MONTERO, LUCERO CAÑEDO-AMAYA, JORGE C. GUILLERMO-HERRERA, JOSSEPH E. UC-VÁZQUEZ, NINA MENDEZ DOMINGUEZ

aspectos de la comunidad donde se está trabajando, saber las expectativas y necesidades, hacer contacto con las instituciones y con la comunidad para motivarlos a participar en la formación de equipos locales auxiliares y en la elaboración de planes de acción integrales con visión a largo plazo ${ }^{2,11}$.Por lo tanto, las facultades de medicina tienen la obligación de dirigir su educación, actividades de investigación y servicios para abordar las preocupaciones de salud de la comunidad, región y/o nación ${ }^{12}$.

\section{Fortalezas en la formación médica}

Entre los aspectos que deben ser evaluados, están las obligaciones y los deberes éticos del médico que deben considerarse en el ámbito comunitario, debido a que el paciente en salud pública involucra a la población y no solo al individuo; los factores socioculturales propios de una región pueden presentarse de forma diferente y variar en principios éticos.

Nuala Kenny y colaboradores nos hablan de la teoría relacional que consiste en: tratar a las personas como seres sociales interrelacionados; los valores que crean la base de este enfoque son la autonomía relacional (a diferencia de la autonomía individual, reconoce que las personas están vinculadas y situadas económica, social y políticamente), justicia social (enfatiza el acceso justo a bienes sociales que incluyen oportunidades y derechos) y solidaridad relacional (inclusión e interconexión colectiva, en lugar de ignorar las diferencias entre personas $)^{13}$.

El paciente, durante su proceso de enfermedad, busca en las instituciones sanitarias un servidor de la salud que le acompañe, ayude, y facilite su recorrido y que además sea de confianza; en la película, el médico Zantoko, requería para sus primeras consultas el ejercicio constante de la comunicación para ganarse la confianza de las personas, por lo que trataba de crear relaciones visitando frecuentemente el bar y así ser acuñado dentro de la comunidad (Foto 2).
En el eje de formación humana el médico debe desarrollar fortalezas y estrategias que le permitan educarse de manera integral, aplicando un conjunto de acciones, comportamientos y motivaciones que lo lleven a realizar actividades altruistas, conservando la integridad y el respeto por los demás ${ }^{14}$. A lo largo del filme se observa como el doctor Zantoko está determinado a alcanzar sus objetivos pese a los obstáculos que enfrenta en la comunidad, en donde predominan costumbres y actitudes conservadoras. Aquellos médicos que adoptan la pasión del humanismo son los que permanecen firmes ante el cumplimiento de sus responsabilidades profesionales a pesar de la adversidad ${ }^{14}$.

En la película se muestra que Zantoko estaba dispuesto a dar prestados sus servicios a cualquier paciente, sin distinción alguna, como a Sylvie, una niña que presentaba problemas dérmicos; su deseo de poder ejercer su profesión lo llevó a investigar su enfermedad para poder dar un diagnóstico y tratamiento adecuado (Foto 3); su formación profesional y humana lo incentivó a dar un trato empático y compasivo valorando las necesidades y autonomía de su paciente ${ }^{15}$.

\section{Estrategias implicadas en la formación del médico en la comunidad}

Como estrategias educativas encontramos enfoques de enseñanza y aprendizaje que se establecen en los planes de estudio y que varían en los modelos curriculares de cada institución. La importancia del diseño del plan de estudios radica en que el alumno de tener un papel de oyente pasa a ser responsable de su propio aprendizaje, de manera que, al evaluar los conocimientos a través de la aplicación de proyectos y ejercicios de autoaprendizaje, la práctica del trabajo colaborativo y el acceso a capacitaciones para el desarrollo de diversas habilidades, le posibilitan visualizar cómo interactuar con una comunidad. En su desarrollo el médico tiene la oportunidad de visitar establecimientos de salud 


\section{LA FORMACIÓN INTEGRAL DEL MÉDICO PARA ENFRENTAR LAS BARRERAS PARA LA ATENCIÓN COMUNITARIA A PROPÓSITO DE EL MÉDICO AFRICANO}

ISAAC E. AGUILAR-CANTÓN, LEYDI G. BURGOS-MONTERO, LUCERO CAÑEDO-AMAYA, JORGE C. GUILLERMO-HERRERA, JOSSEPH E. UC-VÁZQUEZ, NINA MENDEZ DOMINGUEZ

pública, instituciones y organizaciones comunitarias, construyendo de este modo, el significado y la conciencia de la medicina comunitaria. Es de menester considerar y evaluar la seriedad con la que se abordan las visitas, evitando así, que el desinterés sea impedimento para conectar el aprendizaje a partir de la experiencia ${ }^{16}$.

La implementación de programas estudiantiles en las facultades de medicina basadas en el aprendizaje centrado en la comunidad han sido una necesidad a partir del hallazgo de que la praxis médica era orientada al individualismo, la enfermedad y la atención hospitalaria, restándole importancia a la promoción de la salud en la comunidad. La literatura sugiere que los planes de estudios relacionados con las problemáticas sociales fomentan actitudes más positivas en la interacción con los mismos, en comparación con los planes de estudio convencionales ${ }^{17}$.

Los planes de estudios en la medicina deben incluir una formación en la investigación participativa basada en la comunidad, promoción de la salud, prevención de enfermedades y determinantes sociales de la salud, capacitando al estudiante para brindar atención individualizada al paciente desde una perspectiva poblacional ${ }^{18}$.

\section{Discusión}

Se ha analizado desde el enfoque de la medicina preventiva la cinta: El médico africano. Al cavilar acerca de la importancia de conocer las herramientas que nos brinda la medicina comunitaria se obtuvo que desempeña un papel crucial en la formación del médico; un acercamiento a la comunidad permite visualizar las grandes oportunidades para implementar medidas preventivas y promover la salud de las personas que la habitan, además resulta ser pieza clave para favorecer y fortalecer la educación del paciente y por consiguiente la preservación de la salud ${ }^{19}$.

Es imprescindible el papel desempeñado por la medicina comunitaria a lo largo del tiempo, con su creación logró sentar precedentes en salud pública y formar parte del eje de desarrollo estratégico para el control de enfermedades; las estrategias consisten en mecanismos de alerta temprana, investigación aplicada al diagnóstico rápido y tratamiento, así como la vigilancia integral de los agentes causales ${ }^{5}$.

El médico africano refleja la realidad de una sociedad aislada que con sus características particulares, miedo a lo diferente y desconocido, crea fisuras en la atención médico-paciente; aunque el doctor Zantoko es capaz de cambiar los puntos de vista que la comunidad tenía sobre él y su familia, es importante considerar que, con la práctica en actividades de proyectos comunitarios con organizaciones locales, el existir el contacto continuo con el representante clave comunitario, la evaluación de las necesidades y búsqueda de información para realizar intervenciones de salud pública en comunidades francesas durante su formación médica son propuestas que consideramos, le hubiesen permitido abordar sus conocimientos en medicina con más eficacia ${ }^{12}$.

El contraste en las condiciones de trabajo en cuanto al contexto social y cultural en el que el doctor Zantoko se encontraba, del urbanismo a un entorno rural, fue un cambio radical y difícil para él, puesto que, la pertenencia a un grupo social totalmente distinto del que provenía lo hizo tomar medidas que consideraba adecuadas, como hacer a un lado su identidad cultural, y los intereses personales de su familia, intentando encajar ante la negativa (racismo) de los habitantes de Marly-Gomont.

Los obstáculos para combatir el racismo derivan, en primer lugar, en la dificultad de señalarlo ${ }^{19}$; en una escena se observa que el granjero hace un brindis de manera irónica por el nuevo trabajador que contrató, Zantoko (que en ausencia de pacientes que acudan por consultas se vio obligado a realizar una labor independiente a la propia), en referencia al error de normalizar los comportamientos racistas en la comunidad. 


\section{LA FORMACIÓN INTEGRAL DEL MÉDICO PARA ENFRENTAR LAS BARRERAS PARA LA ATENCIÓN COMUNITARIA A PROPÓSITO DE EL MÉDICO AFRICANO}

ISAAC E. AGUILAR-CANTÓN, LEYDI G. BURGOS-MONTERO, LUCERO CAÑEDO-AMAYA, JORGE C. GUILLERMO-HERRERA, JOSSEPH E. UC-VÁZQUEZ, NINA MENDEZ DOMINGUEZ

El médico en comunidad tiene el compromiso de satisfacer las necesidades y problemas de salud de la misma ${ }^{11}$,formar médicos mejor preparados para la realidades fuera de los consultorios implica entregarles las herramientas y las estrategias que en la práctica comunitaria guíen el aprendizaje-servicio, comprometiendo una educación integral en la que se combina el servicio comunitario con los objetivos del aprendizaje de las instituciones educativas, abordando las necesidades en salud eficazmente.

\section{CONCLUSIÓN}

En conclusión, la formación integral del médico debe contar con estrategias óptimas que fomenten fortalezas no solo profesionales sino humanas y éticas, que sean adecuadas en los distintos contextos sociales y culturales en los que se presten servicios sanitarios dentro de una comunidad, con la finalidad de que dichas habilidades le permitan desenvolverse y brindar una atención integral al paciente. Desde una perspectiva docente, el médico africano se puede emplear para el análisis de la labor médica en una comunidad, puesto que ofrece escenas donde se atienden diversos problemas que puede enfrentar un estudiante de medicina e incluso un profesional de la salud.

\section{Referencias}

1. Ruano AL. The role of social participation in municipal-level health systems: the case of Palencia, Guatemala. Glob. Health Action. 2013; 6: 20786.

2. Sharma AK. Community medicine: Beyond boundaries. Indian J Public Health. 2017; 61(2): 65-66.

3. Rosenberg E, Richard C, Lussier MT, Abdool SN. Intercultural communication competence in family medicine: lessons from the field. Patient Educ. Couns. 2006; 61(2): 236-45.

4. Brickner PW. Is there a discipline of community medicine? Am. J. Med. 1976; 60(7): 936-40.
5. Silva Paim J. Medicina comunitaria: Introducción a un análisis crítico. Salud colect. 2009; 5(1): 121-6.

6. Martín ZA. Alma Ata y medicina de familia: 40 años de travesía del desierto. Aten Primaria. 2018; 50(4): 203-4.

7. Sagrado S. Atención primaria en Francia. Semergen. 2016; 42(1): 58-62.

8. Rivenbark JG, Ichou M. Discrimination in healthcare as a barrier to care: experiences of socially disadvantaged populations in France from a nationally representative survey. BMC Public Health. 2020; 20(1): 2-4.

9. Williams DR, Lawrence JA, Davis BA. Racism and Health: Evidence and Needed Research. Annu. Rev. Public Health. 2019; 40: 105-125.

10. Vigneron $E$. Inégalités de santé, inégalités de soins dans les territoires français. Bull Acad. Natl. Med. 2012; 196(4-5): 939-52.

11. Kainuma M, Kikukawa M, Nagata M, Yoshida M. Competencies necessary for becoming a leader in the field of community medicine: a Japanese qualitative interview study. BMJ Open. 2018; Apr 17; 8(4): e020082.

12. Essa-Hadad J, Murdoch-Eaton D, Rudolf MC. What impact does community service learning have on medical students appreciation of population health? Public Health. 2015; 129(11): 1444-1451.

13. Lee LM. Public health ethics theory: review and path to convergence. J. Law Med. Ethics. 2012; 40(1): 85-98.

14. Cohen JJ. Viewpoint: linking professionalism to humanism: what it means, why it matters. Acad. Med. 2007; 82(11): 1029-32.

15. Swick HM. Viewpoint: professionalism and humanism beyond the academic health center. Acad. Med. 2007; 82(11): 1022-28.

16. Dongre AR, Chacko TV. A critical review of new competency-based curriculum for community medicine using various curricular review frameworks. Indian J. Public Health. 2019; 63(4): 362-66.

17. Rolfe IE, Pearson SA, Cleary EG, Gannon C. Attitudes towards community medicine: a comparison of

Rev. Med. Cine. 2021; 17 (3), 187-195 Ediciones Universidad de Salamanca / @®@@ J. Med. Mov., 2021; 17 (3), $187-195$ [ 193 ] 


\section{LA FORMACIÓN INTEGRAL DEL MÉDICO PARA ENFRENTAR LAS BARRERAS PARA LA ATENCIÓN COMUNITARIA A PROPÓSITO DE EL MÉDICO AFRICANO}

ISAAC E. AGUILAR-CANTÓN, LEYDI G. BURGOS-MONTERO, LUCERO CAÑEDO-AMAYA, JORGE C. GUILLERMO-HERRERA, JOSSEPH E. UC-VÁZQUEZ, NINA MENDEZ DOMINGUEZ

students from traditional and community-oriented medical schools. Med. Educ. 1999; 33(8): 606-11.

18. Gimpel N, Kindratt T, Dawson A, Pagels P. Community action research track: Community-based participatory research and service-learning experiences for medical students. Perspect. Med. Educ. 2018; 7(2): 139-43.

19. Cognet M \& Carlier P. (2018) Racism and Healthcare: Representations of the 'Other' in Health Services. SOJ Psychol. 5(2): 1-5.

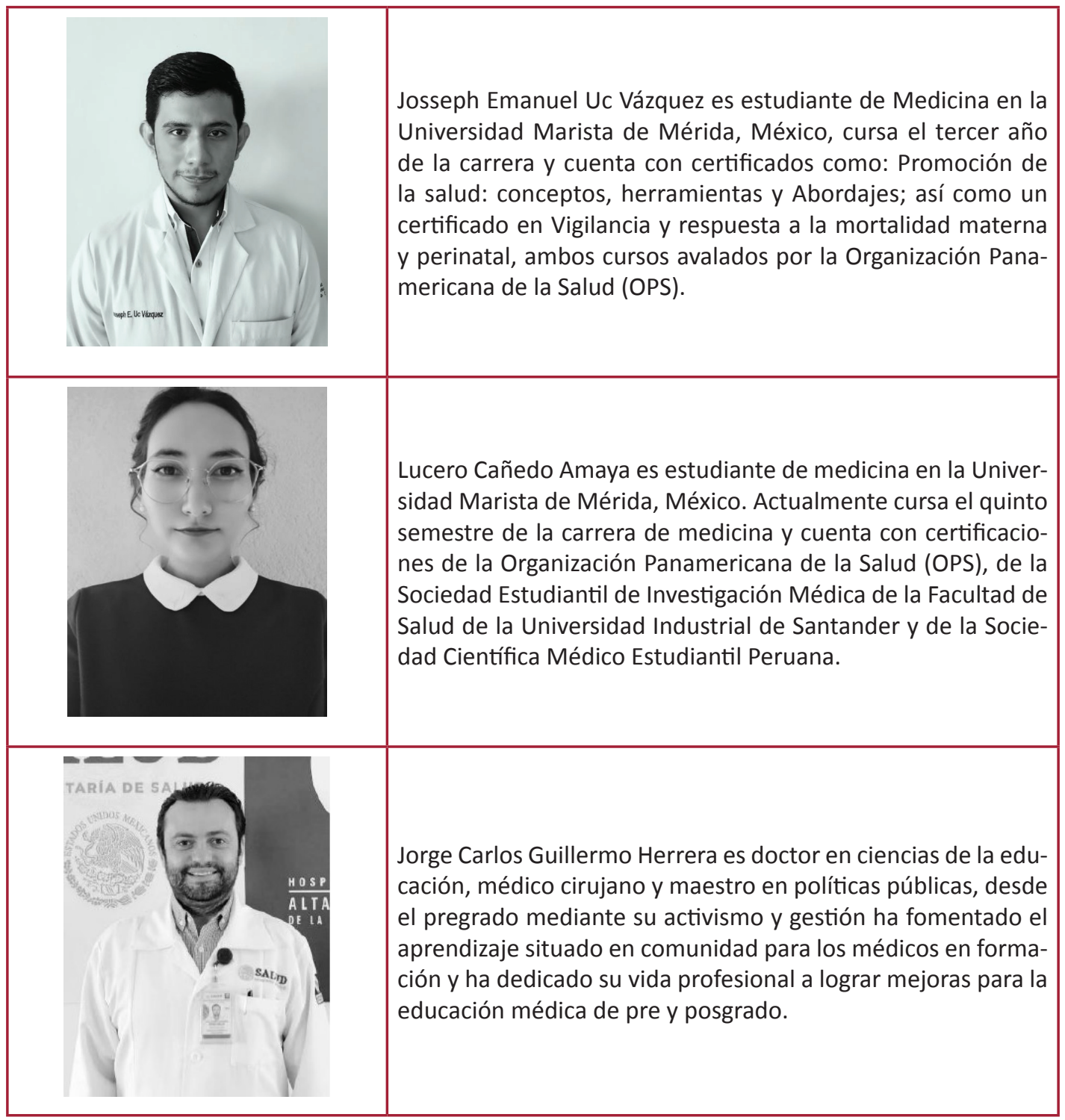




Leydi Guadalupe Burgos Montero es estudiante de la carrera
Médico Cirujano en la Universidad Marista de Mérida, México,
actualmente cursa el quinto semestre. Cuenta con certifi-
caciones de la Organización Panamericana de la Salud, de la
Sociedad Científica Médico Estudiantil Peruana, de la Sociedad
Estudiantil de Investigación Médica de la Facultad de Salud
de la Universidad Industrial de Santander y de la Facultad de
Enfermería de la Universidad Autónoma de Campeche.

